\title{
Diversité et structure des cordons ripicoles le long de la sirba (Nord-Est du Burkina Faso)
}

\author{
Elice KABORE *, Oumarou SAMBARE, Amadé OUEDRAOGO et \\ Adjima THIOMBIANO \\ Laboratoire de Biologie et Ecologie Végétales, Université de Ouagadougou, 03 BP 7021 Ouagadougou 03, \\ Burkina Faso. \\ *Auteur correspondant, E-mail : kabor.elisa@yahoo.fr, Tel :00(226) 71057992 / 76032991
}

\section{RESUME}

Les Cordons ripicoles sont des végétations bordant les rivières à écoulement temporaire et qui possèdent des sols riches et une grande capacité en bois. Ces ressources sont anarchiquement utilisées par l'homme créant ainsi la fragmentation des berges. Cette étude s'inscrit dans le cadre d'une évaluation de la diversité et de la structure des cordons ripicoles en fonction de l'orientation de l'écoulement (amont, zone intermédiaire, aval) et par rapport à la savane adjacente du cours d'eau temporaire la Sirba. L'analyse de 218 relevés dendrométriques ont montré que la richesse spécifique et la diversité sont plus élevées dans les savanes adjacentes. Il existe néanmoins un important potentiel en bois dans les cordons ripicoles révélé par une surface terrière moyenne forte $\left(27,32 \mathrm{~m}^{2} / \mathrm{ha}\right)$ et une densité forte. La richesse spécifique, l'indice d'importance des espèces (IVI), celui d'importance des familles (FIV) et la diversité sont significativement différentes dans les trois positions d'écoulement. La prédominance des Rubiaceae dans la zone intermédiaire traduit une meilleure inondation de ce site. La structure des cordons ripicoles, des savanes adjacentes et des espèces écologiquement importantes est relativement stable. Cependant, la densité faible des individus juvéniles laisse voir un problème de régénération de ces écosystèmes.

(C) 2013 International Formulae Group. All rights reserved.

Mots clés: Etat, Phytodiversité, cours d'eau temporaire, écoulement, dégradation des berges, Sahel.

\section{INTRODUCTION}

Les termes cordon ripicole, forêt ripicole (Guinko, 1984), cordon rivulaire (Mouchet et al., 2004) ont été utilisés pour désigner des végétations de berge dont les espèces constitutives sont adaptées à une submersion plus ou moins longue du système racinaire et même du système aérien en période de crue ; leur existence réside dans la présence de l'eau. Selon Fontès et Guinko (1995) la galerie forestière se distingue nettement des cordons ripicoles par leurs espèces semi-sempervirentes à sempervirentes et qui s'étendent sur les berges des cours d'eau. En région Ouest africaine, Monnier (1990) a décrit les forêts riveraines comme étant des forêts décidues caractérisées par une forme linéaire, une originalité structurale et un microclimat spécifique qui dépendent de l'inondation et du transport des sédiments de la rivière. Mais dans le contexte des pays sahéliens comme le Benin où les savanes sont les écosystèmes les plus dominants, Natta (2003) suggère d'utiliser l'expression 
formation ripicole pour tout type de forêts qui arrive au niveau des rives des fleuves et des rivières. Ces formations comportent donc les forêts semi-décidues, les forêts claires et les savanes situées au niveau des berges des fleuves et des rivières.

Au Burkina Faso, l'expression cordons ripicoles a été utilisée pour désigner de minces cordons de végétation se retrouvant au bord des cours d'eau à écoulement temporaire avec une abondance des espèces buissonnantes les rendant souvent impénétrables (Fontès et Guinko, 1995). Selon Sambaré (2013), les cordons ripicoles sont une végétation rencontrée sur des rives basses et plate rarement inondées et dont les espèces constitutives ne diffèrent généralement pas de celles des milieux adjacents. Elle se rencontre le long des cours d'eau temporaires du domaine sahélien et du secteur nordsoudanien. En opposition, les forêts galeries sont une végétation rencontrée au bord des rivières à écoulement permanent (Guinko, 1984 ; Fontès et Guinko, 1995). Les galeries y sont larges avec une présence importante d'espèces guinéennes. Au Burkina Faso, c'est dans les galeries forestières que l'on trouve des potentialités en bois d'œuvre qui favorisent ou qui pourraient encourager l'installation de scieries.

Les cordons ripicoles sont des écosystèmes diversifiés. Elles constituent généralement un refuge pour les animaux sauvages (Natta, 2003 ; Belem, 2008). Cette végétation protège les berges de l'avènement et de la progression de l'érosion (Hubble et al., 2010), régule la qualité de l'eau, emmagasine les sédiments et contribue à la conservation de la biodiversité (Naiman et al., 2005). Ces écosystèmes de cordons ripicoles abritent d'importantes ressources à savoir des sols humides et fertiles, l'eau, les produits ligneux et non ligneux qui sont utilisés par la population riveraine pour satisfaire leurs besoins de base et comme source de revenus (Natta, 2003).

Cependant, le recul des berges cause la régression des cordons ripicoles (Hien et al., 1995) et cela est accentué par les actions anthropiques qui sont responsables des changements les plus rapides des communautés végétales, même si le facteur climatique est le plus déterminant dans la distribution des espèces (Tremblay et al., 2002). Aussi, la croissance démographique conduit les populations des zones rurales à changer leurs habitudes d'utilisation des terres (Cecchi et al., 2007). C'est ainsi que, l'intensification de la mise en valeur des basfonds (zones de dépression environnantes des cours d'eau) s'est imposée (Lavigne Delville et al., 1996). Parmi les facteurs anthropiques, c'est la construction des barrages qui impacte encore de façon plus significative l'environnement des rivières (Braatne et al., 2007). Classées généralement comme écosystèmes menacés, particulièrement dans les pays sahéliens (Natta 2003) comme le Burkina Faso, les mesures de protection et de restauration de la biodiversité de ces écosystèmes doivent être entreprises. Pour cela, il est nécessaire d'avoir une connaissance fine sur ces formations végétales pour identifier les services éco-systémiques, évaluer la productivité, le carbone séquestré et envisager la protection des espèces vulnérables.

Des études ont permis d'avoir des connaissances sur la flore des cordons ripicoles et sa répartition en fonction des secteurs phytogéographiques et des types de cours d'eau (Sambaré et al., 2011). Les caractéristiques taxonomiques et structurales des cordons ripicoles de certains cours d'eau temporaires ont été abordées par les mêmes auteurs. Cependant, aucune étude n'a été menée spécifiquement sur la Sirba qui est un cours d'eau temporaire situé au Nord-est du Burkina Faso. C'est la rivière la plus importante du secteur phytogéographique sudsahélien. La diversité dépend des secteurs phytogéographiques mais aussi des régimes des cours d'eau (Sambaré, 2013). Mais au sein d'un même cours d'eau la diversité dépend telle de l'orientation de l'écoulement ou des dimensions du lit? Ainsi, les variations que peuvent induire ces deux paramètres sur la composition, la diversité et la structure de la 
végétation des cordons ripicoles selon l'orientation de l'écoulement du cours d'eau, c'est-à-dire qu'elle soit en amont, dans la zone intermédiaire ou en aval sont des aspects importants à étudier.

La répartition des nutriments le long des rivières et par conséquent celle de la végétation, est fonction du flux qui est le composant principal de l'environnement physique (Poff et al., 1997) et du débit d'écoulement du cours d'eau. La diminution du débit conduit à un envasement du lit et la colonisation de la rivière par des espèces moins hygrophiles (Henszey et al., 1991). En effet, l'aval qui est l'embouchure du cours d'eau accumule plus de sédiments, d'éléments chimiques comme le phosphore et le nitrogène que l'amont (Morse et al., 2004).

L'objectif de cette étude est de décrire la composition, d'évaluer la diversité et d'établir la structure des cordons ripicoles en comparaison avec les savanes adjacentes de la Sirba suivant l'orientation de l'écoulement (amont, intermédiaire et aval). L'étude repose sur l'hypothèse selon laquelle, la composition floristique et les paramètres structuraux des cordons ripicoles sont influencés par leur position amont, intermédiaire et en aval du cours d'eau.

\section{MATERIEL ET METHODES \\ Site d'étude}

L'étude s'est déroulée dans la zone climatique soudano-sahélienne du Burkina Faso, le long de la rivière Sirba qui s'étend entre les secteurs phytogéographiques sudsahélien et nord-soudanien (Figure 1). La zone climatique soudano-sahélienne est caractérisée par une pluviométrie annuelle variant entre 600 et $900 \mathrm{~mm}$ avec une seule saison pluvieuse qui dure 4 à 5 mois (Dipama, 2010). La température moyenne annuelle enregistrée par la station météorologique la plus proche (Bogandé) est de $29{ }^{\circ} \mathrm{C}$. La température moyenne de la station de Boulsa est de 28,26 ${ }^{\circ} \mathrm{C}$.

La sirba est un cours d'eau à écoulement temporaire avec une crue au mois de septembre. Elle prend sa source dans la province du Ganzourgou (secteur phytogéographique nord soudanien). Il coule vers le Nord-est et traverse un relief relativement plat à travers les provinces de la Gnagna et de la Komandjari pour se jeter dans le fleuve Niger. Elle à une longueur de 250 km (Dipama, 2010). Le sol des berges a une texture pour la plupart limono-sableuse. Les sites d'études ont été installés en amont, dans la zone intermédiaire et en aval du cours d'eau après une analyse du parcours de la rivière et des prospections de terrain. Ainsi, un site a été retenu dans le département de Boulsa $\left(0^{\circ} 30^{\prime} 8,07^{\prime \prime} \mathrm{W}\right.$ et $\left.12^{\circ} 31^{\prime} 9,952^{\prime \prime} \mathrm{N}\right)$ dans le secteur phytogéographique nord soudanien comme la partie amont. Le site intermédiaire a été installé dans le département de Gayéri $\left(0^{\circ} 24^{\prime} 58,612^{\prime \prime} \mathrm{E}\right.$ et $\left.12^{\circ} 466^{\prime} 35,903^{\prime \prime} \mathrm{N}\right)$ dans le secteur phytogéographique sub-sahélien Dans la partie aval, le site a été installé dans le département de Bartiébougou (049'15,809"E et $\left.12^{\circ} 50^{\prime} 41,261^{\prime \prime} \mathrm{N}\right)$. Ce département est également situé dans le secteur phytogéographique sub-sahélien et fait frontière avec le Niger. La plupart des villages riverains des sites d'étude sont des hameaux de culture au niveau desquels les éleveurs et les agriculteurs se sont installés pour profiter de la proximité de l'eau et des ressources des cordons ripicoles.

Une culture maraîchère s'est développée le long de la rivière et concerne principalement le tabac, le haricot sucré et la pastèque. Elle se pratique même dans le lit de la rivière après la décrue.

Dans l'ensemble de la zone parcourue par la Sirba, la végétation est dominée par la savane à épineux dont les espèces communes sont Balanites aegyptiaca, Acacia senegal, Acacia seyal, Acacia gourmaensis, Ziziphus mauritiana. Quant aux cordons ripicoles, ils sont principalement tributaires de la Sirba. Ils ont une physionomie dense avec une prédominance de Mitragyna inermis, Acacia ataxacantha, Acacia pennata, Fluggea virosa, Rytigynia senegalensis, Crataeva adansonii, Ziziphus spina-christi, Piliostigma reticulatum Dyospyros mespiliformis (Fontès et Guinko, 1995). 


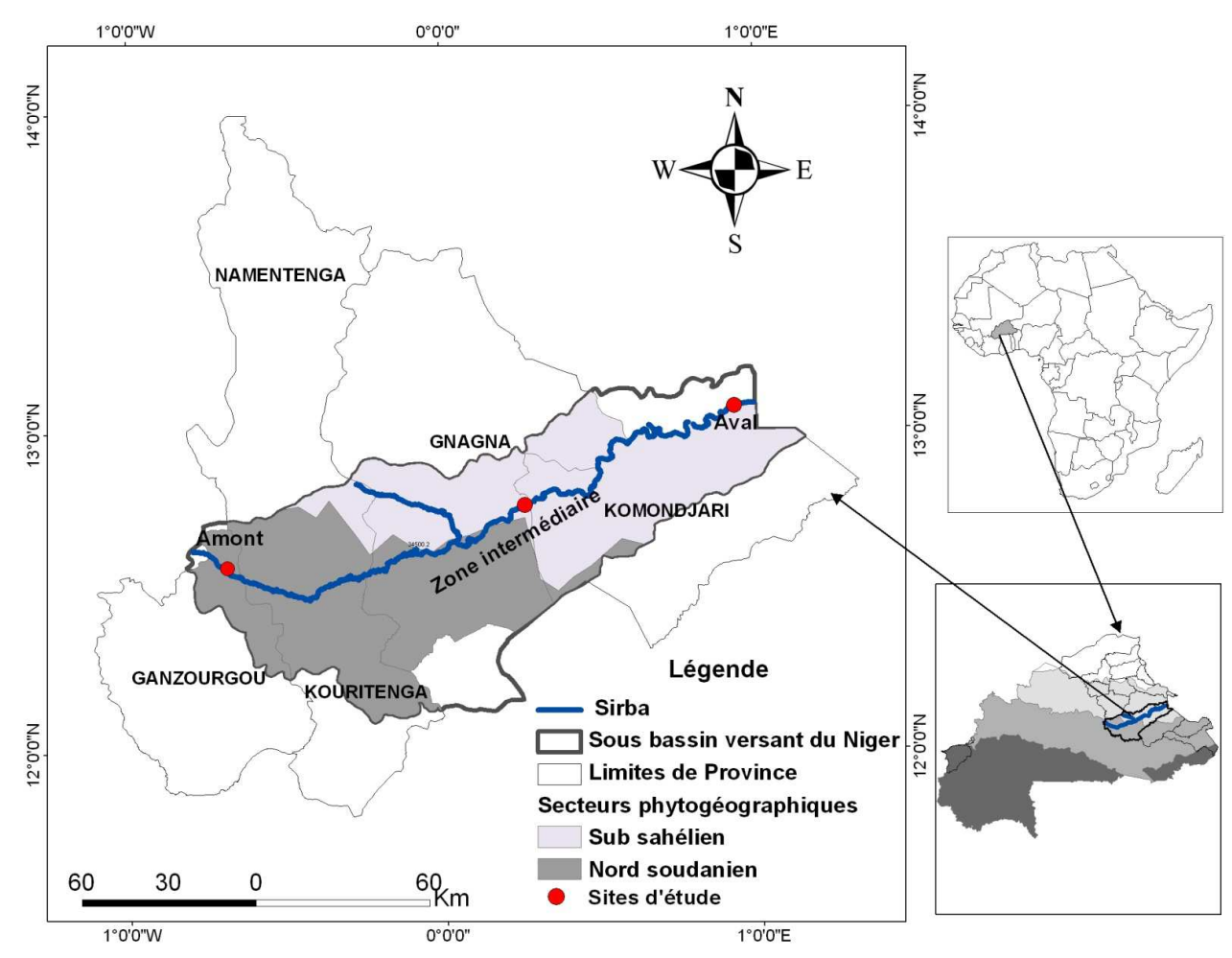

Figure 1: Localisation des sites d'étude le long de la Sirba.

\section{Collecte des données}

Les données ont été collectées en amont, dans la zone intermédiaire et en aval de la rivière en Octobre-Novembre 2011 et Septembre-Novembre 2012. Dans cette étude, l'amont représente la source évidente du fleuve et l'aval représente l'embouchure dans les limites du territoire Burkinabé. Quant à la zone intermédiaire c'est la zone de miparcours de la rivière. Sur chaque site au minimum 30 transects perpendiculaires au cours d'eau principal ont été installés séparés entre eux par des intervalles de $200 \mathrm{~m}$. Sur chaque transect, une placette est installée dans la savane adjacente et une autre dans les cordons ripicoles, de part et d'autre du cours d'eau. Les superficies des placettes sont de $500 \mathrm{~m}^{2}(50 \mathrm{~m} \mathrm{X} 10 \mathrm{~m})$ dans les cordons ripicoles (Natta, 2003 ; Sokpon et al., 2001) et de $1000 \mathrm{~m}^{2}(50 \mathrm{~m}$ X $20 \mathrm{~m})$ dans les formations adjacentes (Ouédraogo et al., 2008) pour l'inventaire de la végétation adulte qui sont les individus dont le diamètre à hauteur de poitrine (dbh) est supérieur ou égal à $5 \mathrm{~cm}$. Dans les placettes de $500 \mathrm{~m}^{2}$ et de $1000 \mathrm{~m}^{2}$, deux sous-placettes de $25 \mathrm{~m}^{2}(5 \mathrm{~m} \mathrm{X}$ $5 \mathrm{~m}$ ) ont été installées pour faire l'inventaire de la régénération qui sont les individus dont le diamètre à hauteur de poitrine (dbh) est inférieur à $5 \mathrm{~cm}$. Un total de 218 relevés dendrométriques a été installé dans les 3 sites dont 137 dans les cordons ripicoles et 81 relevés dans les savanes (Tableau 6). Le nombre de relevés dans les cordons ripicoles diffère de ce des savanes en raison de l'absence de la savane par endroit.

\section{Paramètres mesurés}

Tous les individus de diamètre supérieur ou égal à $5 \mathrm{~cm}$ à $1,30 \mathrm{~m}$ du sol sont considérés comme des adultes et le reste est rangé dans la régénération. Pour les adultes les paramètres mesurés sont: le diamètre à $1,30 \mathrm{~m} \mathrm{du}$ sol de toutes les tiges, la hauteur 
totale et le nombre de tiges par individu. Pour les individus multicaules le diamètre quadratique est calculé (Dq) selon la formule suivante :où di= diamètre de chaque tige

$$
D q=\sqrt{\sum_{i=1}^{n}\left(d i^{2}\right)}
$$

L'inventaire de la régénération a consisté à mesurer la hauteur totale de tous les individus juvéniles (semis, drageons, rejets de souches) et le diamètre au collet.

\section{Traitement des données}

Les paramètres suivants ont été utilisés pour la caractérisation des peuplements et l'analyse floristique.

$$
\begin{aligned}
& \text { Surface terrière }=\mathrm{D}_{1,30}^{2} \frac{\pi}{4}(2) \\
& \mathrm{D} \mathrm{R}=\frac{\text { Surface terrièr e totale d' une espèce }}{\text { Surface terrière totale de toutes les espèces }} \times 100 \\
& \mathrm{DR}=\text { Dominance relative } \\
& \text { Densité relative }=\frac{\text { Nombred'individusd'uneespèce }}{\text { Nombretotal d'individus }} \times 100 \\
& \text { Diversité relative }=\frac{\text { Nombre d'espèces d'unefamille }}{\text { Nombre total d'espèces }} \times 100
\end{aligned}
$$

$$
\text { Fréquence }=\frac{\text { Nombre de relevés dans lequel l'espèce est présente }}{\text { Nombre total de relevés }} \times 100
$$$$
\text { Fréquence relative }=\frac{\text { Fréquence d'une espèce }}{\text { Somme de toutes les fréquences }} \times 100
$$

L'indice de valeur d'importance (IVI) = dominance relative + densité relative + fréquence relative (3);

Valeur d'importance de la famille (FIV) $=$ dominance relative + densité relative + diversité relative (4), où $\mathrm{D}_{1,30}$ est le diamètre $\mathrm{du}$ tronc à $1,30 \mathrm{~m}$ du sol. Théoriquement la dominance relative, la densité relative, la fréquence relative et la diversité relative varient de 0 à $100 \%$, ainsi l'IVI des espèces et la FIV doivent varier de 0 à $300 \%$.
Des indices de diversité dont celui de Shannon $(\mathrm{H})$ et de Piélou $\left(\mathrm{E}_{\mathrm{H}}\right)$ ont été calculés pour mieux rendre compte de la diversité et de la composition des communautés végétales. Ils fournissent plus d'informations sur la composition par rapport à la simple richesse spécifique. Ils prennent en compte les abondances relatives des différentes espèces. La richesse spécifique est souvent insuffisante pour comparer deux communautés parce qu'elle ne prend pas en compte la dominance relative de chaque espèce (Krebs, 1999). En prenant en compte l'abondance relative, un indice de diversité ne dépend pas seulement de la richesse spécifique mais aussi de la façon dont les individus sont distribués dans les peuplements.

$\mathrm{H}=-\sum_{i=1}^{S} p_{i} \ln p_{i}(5) \quad \mathrm{E}_{\mathrm{H}}=\mathrm{H} / \ln S$

où $\mathrm{S}=$ nombre total des espèces dans une communauté (richesse) et $p i=1$ 'abondance relative de la ième espèces dans une placette. Ces indices sont largement utilisés pour mesurer la diversité biologique (Magurran, 2004). L'indice de diversité de Shannon (H) prend en compte la richesse spécifique et l'équitabilité. Il croît avec le nombre d'espèces dans la placette et la diversité maximale est égale au nombre d'espèce dans l'écosystème. L'équitabilité de Piélou mesure l'abondance relative des différentes espèces constituant la richesse d'une aire. Cela suppose une valeur évoluant entre 0 et 1 avec 1 étant l'équitabilité maximale. Pour évaluer la similarité entre cordons ripicoles et les savanes adjacentes, l'indice de similarité de Sørensen a été calculé sur la base de la présence/absence des espèces.

Indice de Sørensen $(\mathrm{Cs})=\frac{2 j}{2 \mathrm{j}+a+b}$

où, $j=$ nombre d'espèces communes aux sites, $a=$ nombre d'espèces trouvées seulement dans le site 1 et $b=$ le nombre d'espèces trouvées dans le site 2. Cet indice varie potentiellement entre 0 et 1 , et une valeur proche de 1 indique une forte similarité entre des sites (Maguran, 2004). Le coefficient de 
similarité de Sørensen est sensible aux différences des dimensions des placettes.

Les caractéristiques structurales (densité de tiges, surface terrière, diamètre moyen) ont été calculées pour chaque placette et les moyennes ont été calculées par unité de végétation.

La richesse spécifique et la densité des populations ont été analysées à l'aide de modèles linéaires généralisés utilisant une quasi-probabilité pénalisée avec des erreurs de type Poisson. Les positions d'écoulement et le type de climat ont été utilisés comme des facteurs catégoriques fixes et randomisés afin de rendre compte de la variabilité naturelle dans l'occurrence de l'espèce suivant ces positions. Les modèles linéaires généralisés avec des erreurs de type Poisson ont été utilisés pour le diamètre à $1,30 \mathrm{~m}$ afin de tenir compte de la distribution non-normale des erreurs et les écarts de plus en plus élevés des moyennes qui sont généralement associés avec des données impliquant des décomptes. L'estimation de la quasi-probabilité pénalisée a été utilisée afin de pallier les amplitudes de dispersion (Crawley, 2005). L'indice de diversité de Shannon a été analysé par le modèle statistique linéaire avec les mêmes facteurs catégoriques fixes et randomisés. Ces caractéristiques structurales remplissent les postulats de normalité et d'homogénéité de la variance. Toutes les analyses statistiques ont été effectués dans le logiciel de statistique Xlstat. Les indices de diversité ont été calculés dans le logiciel Pcord 6. Les calculs de similarité ont été effectués dans le logiciel CAP.

Les structures de distribution des populations et des espèces dominantes ont été ajustées au modèle de Weibull à 3 paramètres dans le logiciel Minitab. Les paramètres utilisés sont "échelle" qui est celui de l'échelle position imposé par le logiciel, "Thresh" le paramètre de la position liée à la valeur centrale des classes et enfin "Shape" qui est celui de la forme noté $\mathrm{C}$ généralement, c'est le plus important des paramètres. En effet, $\mathrm{C}$ est le paramètre de forme lié à la structure observée, il indique en fonction de sa valeur les conditions de vie du peuplement.

\section{RESULTATS \\ Diversité floristique}

Un total de 99 espèces ligneuses ont été recensées et repartis en 69 genres et 33 familles sur l'ensemble des sites. Parmi ces espèces, 70 ont été recensées dans les cordons ripicoles dont 39 dans la partie amont, 41 dans la partie intermédiaire et 47 dans la partie aval. Les savanes renferment 85 des 99 espèces dont 65 d'entre elles ont été retrouvées dans la partie amont, 51 dans la partie intermédiaire et 47 dans la partie en aval (Tableau 8).

La richesse spécifique moyenne ligneuse des cordons ripicoles est plus élevée dans la zone intermédiaire $(8,97 \pm 0,62$ espèces par $500 \mathrm{~m}^{2}$ ) (Tableau 1). Celle des savanes adjacentes est plus élevée en amont $\left(13,26 \pm 0,77\right.$ espèces par $\left.1000 \mathrm{~m}^{2}\right)$ et décroit jusqu'en aval (Tableau 2). Cependant, celle des adultes (dbh $\geq 5 \mathrm{~cm})$ varie significativement d'une position d'écoulement à une autre dans les cordons ripicoles (Tableau 2). La richesse spécifique moyenne est plus élevée en aval (3,63 $\pm 0,29$ espèces $\left.1500 \mathrm{~m}^{2}\right)$ et plus faible en amont $(2,5 \pm 0,22$ espèces par $500 \mathrm{~m}^{2}$ ). Dans les savanes adjacentes, la richesse spécifique moyenne est plus élevée avec une différence significative $(\mathrm{P}<$ 0,012) dans la zone intermédiaire $\left(5,14 \pm 0,57\right.$ espèces par $\left.1000 \mathrm{~m}^{2}\right)$ (Tableau 1) et plus faible en amont.

Les Indices des Valeurs d'Importance (IVI) des espèces varient en fonction des positions dans chaque type de formation (Tableau 3). Dans les cordons ripicoles, les espèces dominantes en fonction des trois positions d'écoulement sont Mitragyna inermis, Piliostigma reticulatum, Anogeissus leiocarpa, Albizia chevalieri et Acacia seyal, en amont; Mitragyna inermis, Piliostigma reticulatum, Diospyros mespiliformis, Daniellia oliverii et Acacia sieberiana dans la 
partie intermédiaire et Mitragyna inermis, Diospyros mespiliformis, Morelia senegalensis, Piliostigma reticulatum, et Feretia apodanthera dans la partie en aval. Dans les savanes, les espèces dominantes en fonction des positions d'écoulement des cordons ripicoles associées sont Balanites aegyptiaca, Vitellaria paradoxa, Acacia seyal, Acacia macrostachya, Acacia nilotica et Acacia gourmaensis dans la partie amont, Balanites aegyptiaca, Piliostigma reticulatum, Mitragyna inermis et Diospyros mespiliformis dans la partie intermédiaire et Combretum glutinosum, Diospyros mespiliformis, Balanites aegyptiaca, Combretum micranthum, Combretum nigricans, Piliostigma reticulatum et Daniellia oliveri dans la partie aval.

Les Indices d'Importance des Familles varient également en fonction des positions d'écoulement suivant les types de formation (Tableau 4). Dans les cordons ripicoles, ce sont les Rubiaceae qui sont la famille la plus importante sur les 3 positions suivies par les Fabaceae les Combretaceae et les Ebenaceae. Par contre dans les savanes ce sont les Fabaceae qui sont la famille la plus importante suivies par les Combretaceae, les Zygophylaceae et les Ebenaceae.

\section{Diversité spécifique et similarité comparées des positions}

La diversité ligneuse globale des cordons ripicoles est plus forte dans la zone intermédiaire et celle des savanes adjacentes est plus forte en amont (Tableau 1). L'indice de diversité de Shannon varie d'une position d'écoulement à une autre dans les cordons ripicoles et dans les savanes adjacentes pour les ligneux adultes $(\mathrm{dbh} \geq 5 \mathrm{~cm})$ (Tableau 2). $\mathrm{Au}$ sein des cordons ripicoles, la plus forte diversité $(1,96 \pm 0,01)$ est observée en aval suivie de près par la zone intermédiaire $(1,67$ $\pm 0,02)$ et l'aval $(1,62 \pm 0,008)$. Par contre dans les savanes, c'est plutôt dans la partie intermédiaire que la plus forte diversité est observée $(2,80 \pm 0,015)$ suivie par l'amont
$(2,67 \pm 0,002)$ et la zone intermédiaire $(2,25 \pm$ $0,002)$. Seule la partie en aval présente une assez bonne équi-répartition avec E $(0,59)$ au niveau des cordons ripicoles. Tous les trois sites des savanes présentent une bonne équirépartition avec des valeurs de $\mathrm{E}$ respectivement de 0,$8 ; 0,79$; et 0,77 .

Selon l'indice de similarité de Sørensen, il existe une grande similarité $(\mathrm{Cs}=0,72)$ entre la flore des cordons ripicoles et celle de la savane adjacente. Les cordons ripicoles des trois positions d'écoulement se ressemblent mais la similarité est plus forte entre l'amont et l'aval (Indice de Sørensen = 0,654) (Tableau 5). Au niveau des savanes les trois positions sont similaires avec une plus forte similarité entre la zone intermédiaire et l'aval (Indice de Sørensen = 0,615) (Tableau 6).

La largeur de la rivière n'est corrélée ni à la densité ni à la richesse spécifique (Tableau 7). En aval la profondeur de la rivière est très faiblement corrélée $\left(\mathrm{R}^{2}=0,151\right)$ à la richesse spécifique (Tableau 7). La largeur de la rivière augmente de l'amont à l'aval mais la profondeur ne suit pas cette même logique (Tableau 7). La zone intermédiaire a la plus grande profondeur moyenne $(\mathrm{P}=3,89)$ et l'amont la plus petite $(\mathrm{p}=1,33)$.

\section{Structure}

Les densités moyennes au niveau des cordons ripicoles varient suivant les trois positions d'écoulement le long du cours d'eau (Tableau 6). La plus forte densité (1226,92 \pm 82,94 individus . ha ${ }^{-1}$ ) est observée en amont et la plus faible densité $(765,106 \pm 87,26$ individus. $\mathrm{ha}^{-1}$ ) en aval. Dans les savanes adjacentes les densités varient également en fonction des positions par rapport au cours d'eau (Tableau 6). La plus forte densité $\left(232,38 \pm 33,50\right.$ individus. ha $\left.^{-1}\right)$ est observée au niveau de la zone intermédiaire et la plus faible densité $\left(107,35 \pm 26,33\right.$ individus. $\left.\mathrm{ha}^{-1}\right)$ en amont. 
Les surfaces terrières varient également en fonction des trois positions topologiques le long du cours d'eau dans les cordons ripicoles (Tableau 6). La plus forte surface terrière $\left(31,07 \pm 2,41 \mathrm{~m}^{2} \cdot\right.$ ha $\left.^{-1}\right)$ a été trouvée en aval et la plus faible dans la zone intermédiaire $\left(24,66 \pm 2,58 \mathrm{~m}^{2} \cdot \mathrm{ha}^{-1}\right)$. Au niveau des savanes adjacentes, il n'y a pas de différences significatives pour la surface terrière entre les trois positions d'écoulement $(\mathrm{P}<0,063)$; Tableau 6). La surface terrière est plus élevée dans la zone intermédiaire (7,43 $\pm 0,73$ $\left.\mathrm{m}^{2} \cdot \mathrm{ha}^{-1}\right)$ suivie par l'aval $\left(4,85 \pm 0,65 \mathrm{~m}^{2} \cdot \mathrm{ha}^{-1}\right)$ et l'amont $\left(2,41 \pm 0,57 \mathrm{~m}^{2} \cdot \mathrm{ha}^{-1}\right)$.

La distribution en classe de diamètre des individus montre une bonne structure en général (Figure 2 et 3 ). Les paramètres de forme inférieurs à 1 indiquent des peuplements jeunes mais avec une mortalité des individus les plus jeunes. Les individus juvéniles sont assez bien distribués dans les différentes classes de hauteur dans les cordons ripicoles et dans les savanes (Figure 4 et 5). Cependant il y a une difficulté des classes inférieures d'atteindre les classes de hauteur et de diamètre élevées.

Quant à la structure des espèces importantes dominantes elle montre dans les cordons ripicoles des tendances en $\mathbf{J}$ renversé avec cependant une grande différence entre la classe [5 10[ et les autres classes de diamètre (Figure 6). La distribution des individus des espèces dominantes des savanes adjacentes montre une tendance en $\mathbf{J}$ renversé pour tous les sites avec une meilleure distribution en aval pour Combretum glutinosum qui imprime la physionomie de cette végétation (Figure 7).
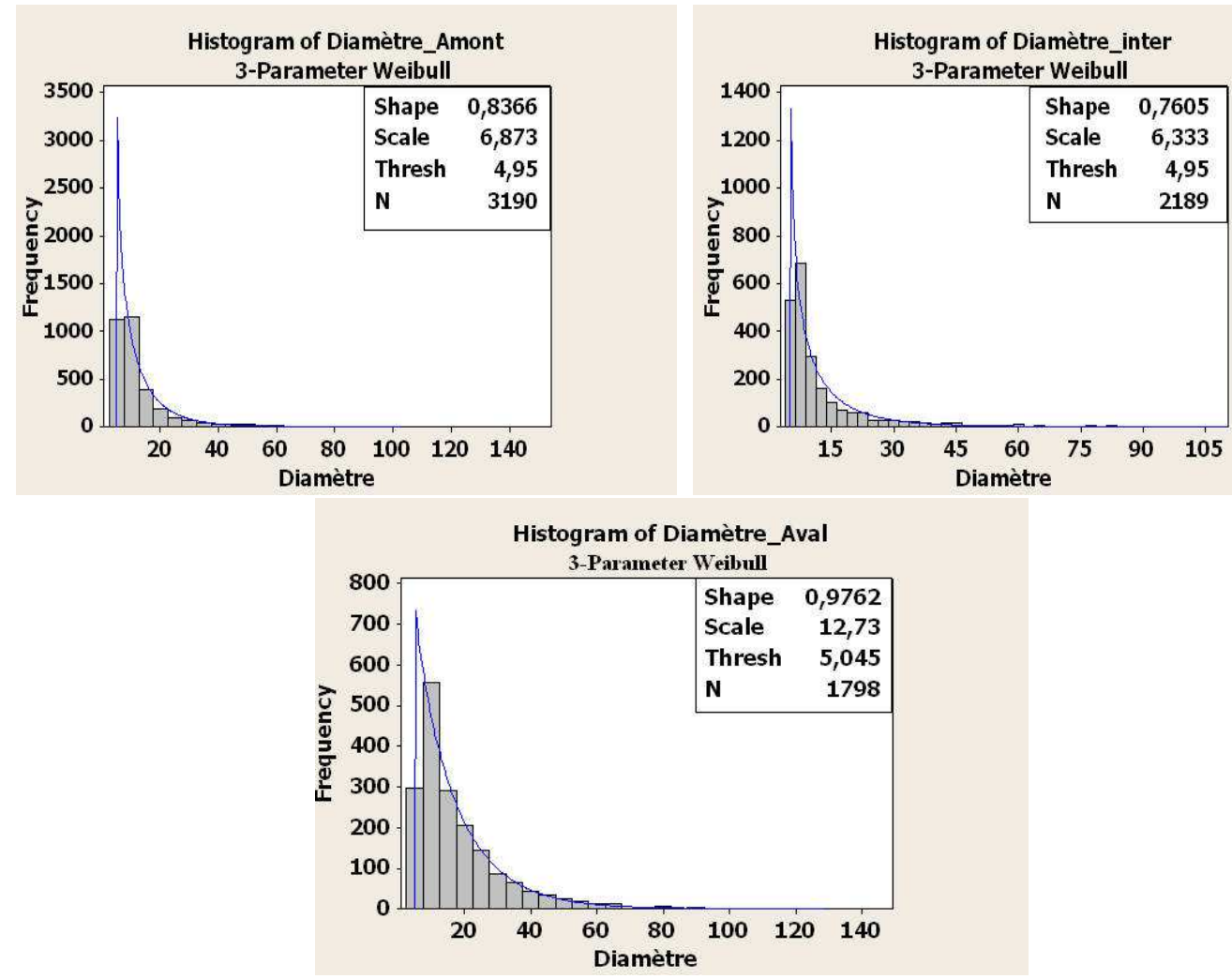

Figure 2 : Structure en classes de diamètre des cordons ripicoles de la Sirba selon leur position par rapport au sens d'écoulement. 

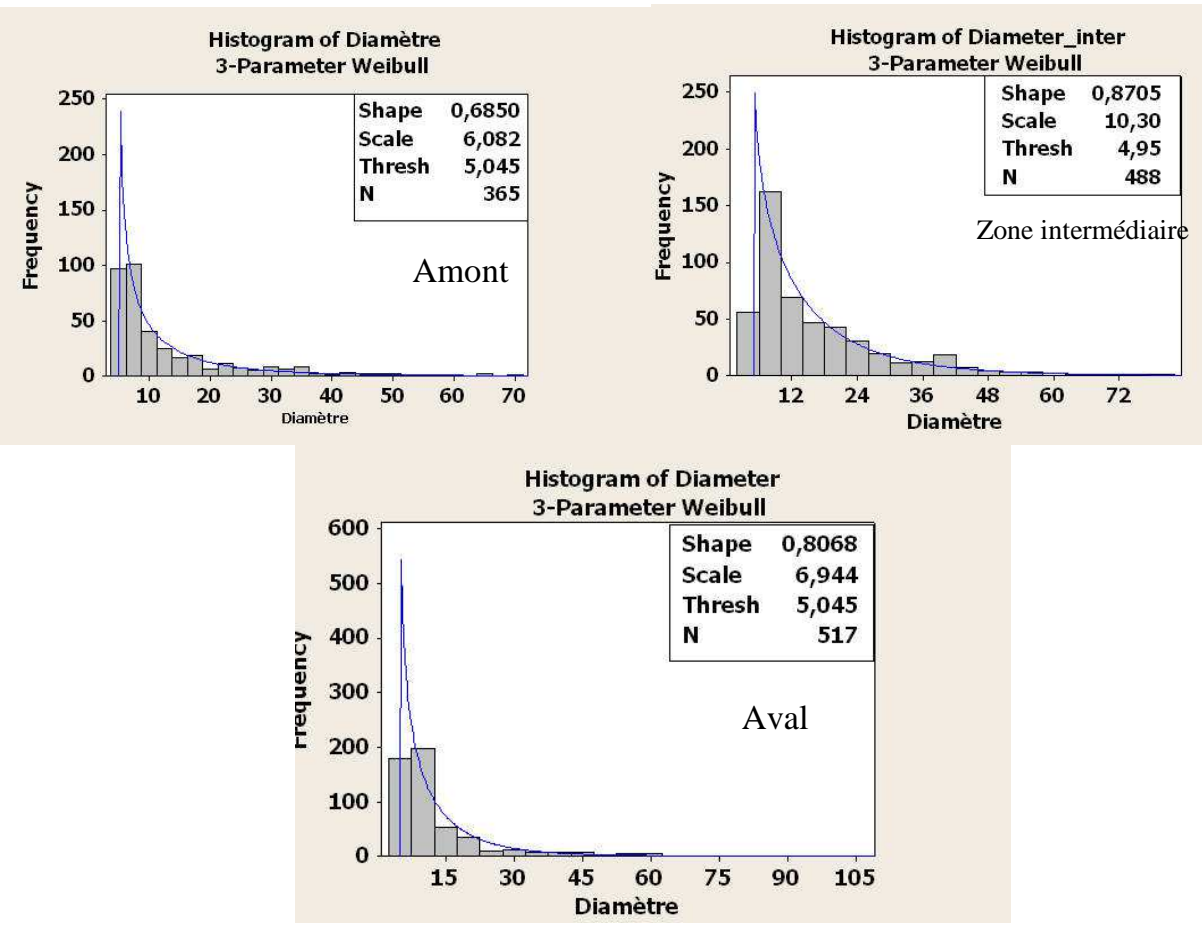

Figure 3 : Structure en classes de diamètre des savanes adjacentes de la Sirba selon leur position par rapport au sens d'écoulement.

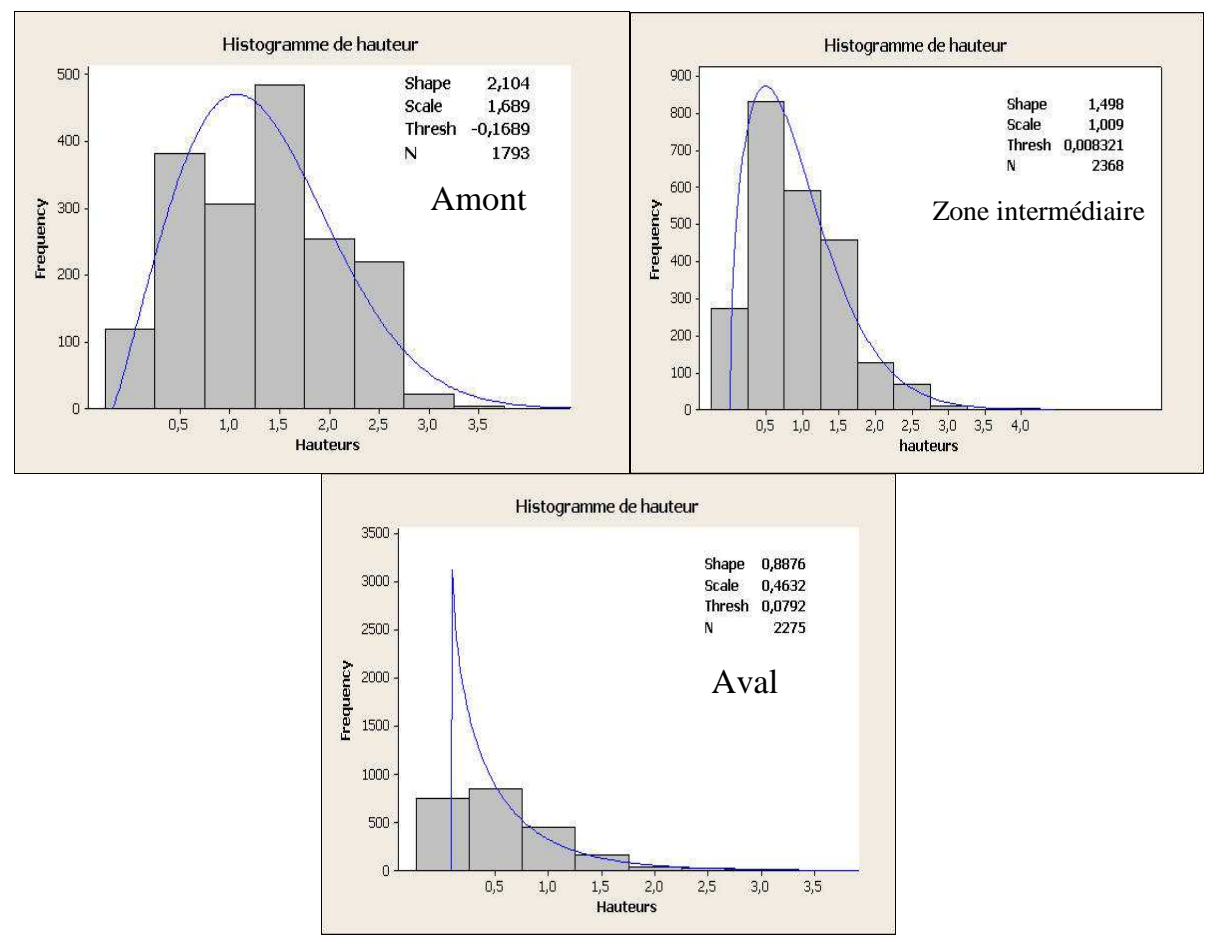

Figure 4 : Distribution des individus juvéniles des cordons ripicoles en classe de hauteur Savanes. 


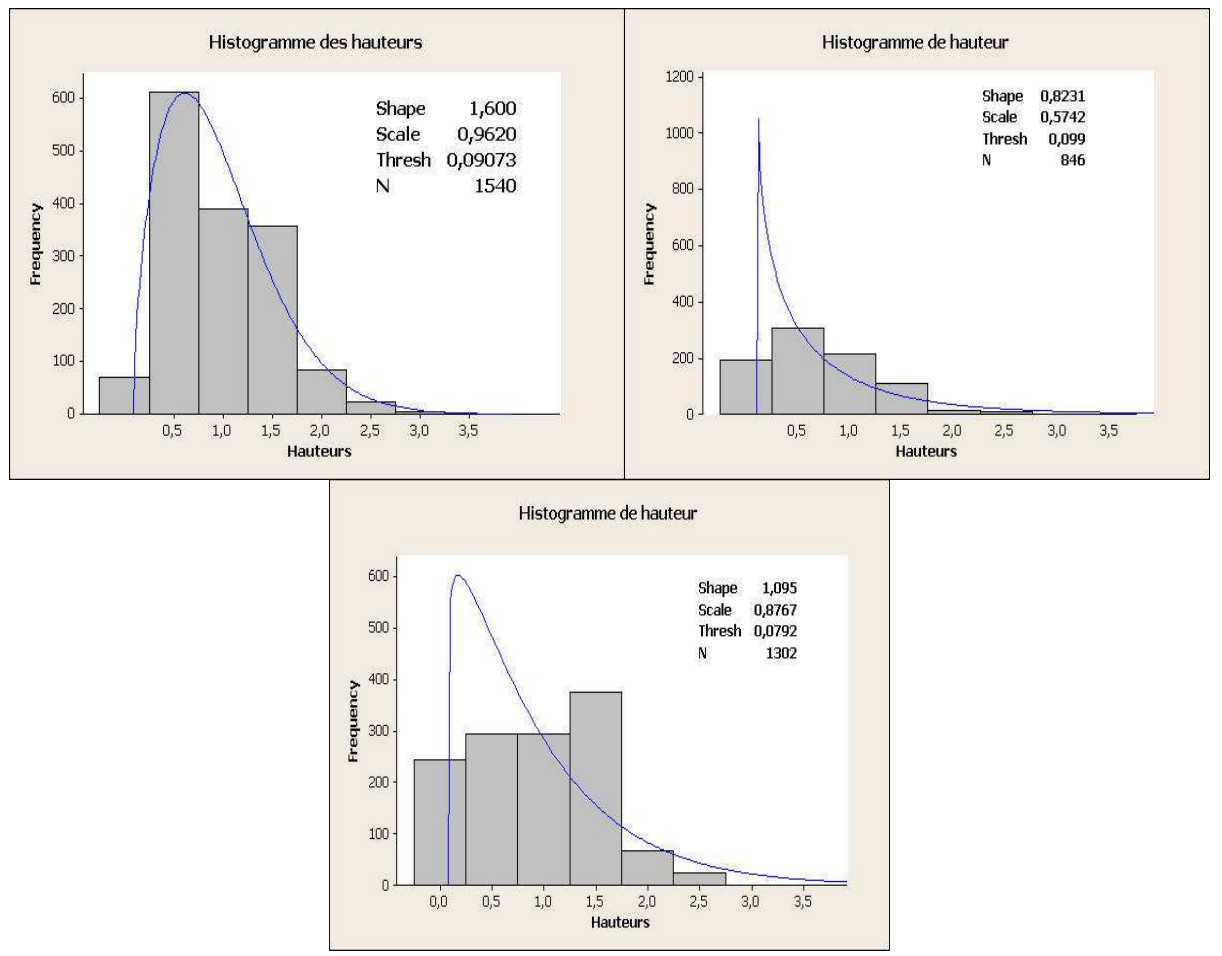

Figure 5 : Distribution des individus juvéniles des savanes adjacentes en classe de hauteur.

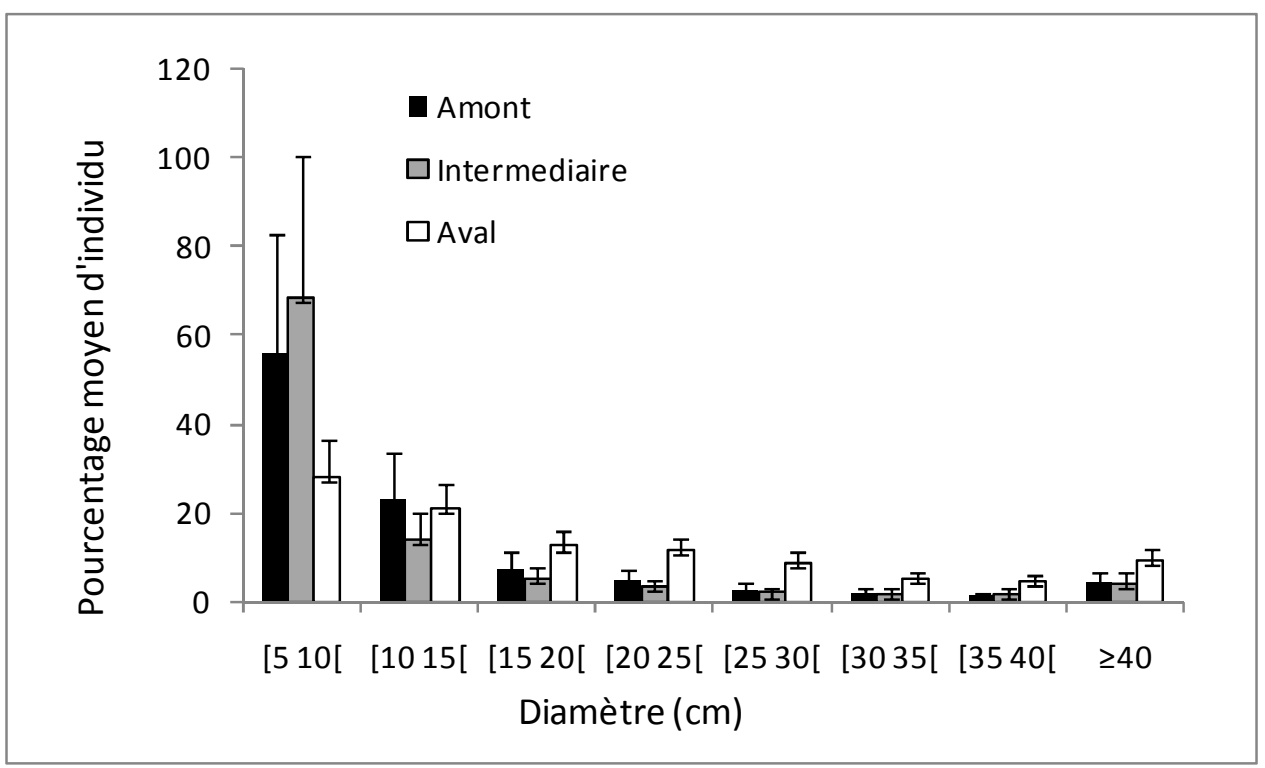

Figure 6: Structure de l'espèce importante (Mitragyna inermis) des 3 sites au niveau des cordons ripicoles. 


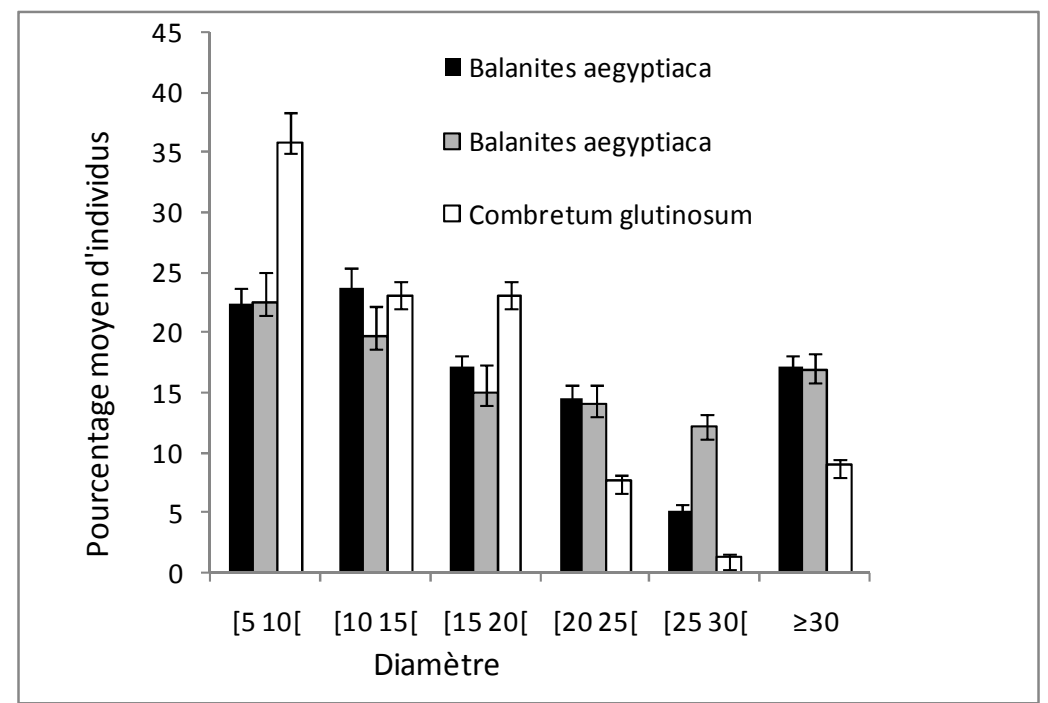

Figure 7: Structure des espèces importantes des savanes adjacentes Balanites aegyptiaca en amont et dans la zone intermédiaire et Combretum glutinosum en aval.

Tableau 1 : Indice de diversité ligneuse globale des cordons et des savanes adjacentes suivant les trois positions d'écoulement le long de la Sirba.

\begin{tabular}{lccccccc}
\hline $\begin{array}{l}\text { Position } \\
\text { géographique }\end{array}$ & $\begin{array}{c}\text { Type de } \\
\text { formations }\end{array}$ & $\begin{array}{c}\text { Nombre } \\
\text { de } \\
\text { familles }\end{array}$ & $\begin{array}{c}\text { Nombre } \\
\text { de genres }\end{array}$ & $\begin{array}{c}\text { Nombre } \\
\text { d'espèces }\end{array}$ & $\begin{array}{c}\text { Richesse } \\
\text { spécifique } \\
\text { moyenne }\end{array}$ & $\begin{array}{c}\text { Indice de } \\
\text { Shannon }\end{array}$ & $\begin{array}{c}\text { Indice } \\
\text { de Piélou }\end{array}$ \\
\hline Amont & Cordon ripicole & 17 & 28 & 40 & $6,47 \pm 0,56$ & $2,05 \pm 0,01$ & 0,548 \\
& Savane & 24 & 42 & 51 & $13,26 \pm 0,77$ & $3,08 \pm 0,008$ & 0,8 \\
\hline Intermédiaire & Cordon ripicole & 20 & 36 & 51 & $8,97 \pm 0,62$ & $1,97 \pm 0,008$ & 0,504 \\
& Savane & 20 & 41 & 61 & $12,28 \pm 1,16$ & $3,02 \pm 0,015$ & 0,794 \\
\hline Aval & Cordon ripicole & 17 & 33 & 48 & $7,76 \pm 0,59$ & $2,09 \pm 0,01$ & 0,546 \\
& Savane & 20 & 37 & 65 & $10,78 \pm 0,60$ & $2,59 \pm 0,01$ & 0,77 \\
\hline
\end{tabular}

Tableau 2 : Indices de diversité des ligneux adultes des cordons ripicoles et des savanes adjacentes suivant trois positions d'écoulement le long de la Sirba.

\begin{tabular}{|l|c|c|c|c|c|c|c|}
\hline $\begin{array}{l}\text { Position } \\
\text { géographique }\end{array}$ & $\begin{array}{c}\text { Type de } \\
\text { formations }\end{array}$ & $\begin{array}{c}\text { Nombre de } \\
\text { familles }\end{array}$ & $\begin{array}{c}\text { Nombre } \\
\text { de genres }\end{array}$ & $\begin{array}{c}\text { Nombre } \\
\text { d'espèces } \\
\text { adultes }\end{array}$ & $\begin{array}{c}\text { Richesse } \\
\text { spécifique } \\
\text { moyenne }\end{array}$ & $\begin{array}{c}\text { Indice de } \\
\text { Shannon }\end{array}$ & $\begin{array}{c}\text { Indice } \\
\text { de Piélou }\end{array}$ \\
\hline Amont & Cordon ripicole & 16 & 19 & 24 & $2,5 \pm 0,22$ & $1,62 \pm 0,01$ & 0,51 \\
\cline { 2 - 8 } & Savane & 17 & 20 & 26 & $3,53 \pm 0,33$ & $2,61 \pm 0,002$ & 0,8 \\
\hline \multirow{2}{*}{ Intermédiaire } & Cordon ripicole & 19 & 23 & 30 & $3,45 \pm 0,24$ & $1,67 \pm 0,02$ & 0,50 \\
\cline { 2 - 8 } & Savane & 20 & 21 & 35 & $5,14 \pm 0,57$ & $2,80 \pm 0,002$ & 0,794 \\
\hline \multirow{2}{*}{ Aval } & Cordon ripicole & 17 & 25 & 29 & $3,63 \pm 0,29$ & $1,96 \pm 0,008$ & 0,59 \\
\cline { 2 - 8 } & Savane & 16 & 26 & 20 & $3,61 \pm 0,22$ & $2,25 \pm 0,015$ & 0,77 \\
\hline
\end{tabular}


Tableau 3 : Liste des espèces de $\mathrm{DBH} \geq 5 \mathrm{~cm}$ recensées avec leur Indice de Valeur d'Importance (IVI).

\begin{tabular}{|c|c|c|c|c|}
\hline \multirow[t]{2}{*}{ zones } & \multicolumn{2}{|c|}{ Cordons ripicoles } & \multicolumn{2}{|c|}{ Savanes adjacentes } \\
\hline & Espèces & IVI & Espèces & IVI \\
\hline \multirow{10}{*}{ Amont } & Mitragyna inermis & 220,84 & Balanites aegyptiaca & 64,52 \\
\hline & Piliostigma reticulatum & 21,47 & Vitellaria paradoxa & 59,83 \\
\hline & Anogeissus leiocarpa & 14,81 & Acacia seyal & 24,35 \\
\hline & Albizia chevalieri & 6,96 & Acacia macrostachya & 23,79 \\
\hline & Acacia seyal & 5,91 & Acacia nilotica & 16,76 \\
\hline & Acacia pennata & 4,1 & Acacia gourmaensis & 15,66 \\
\hline & Acacia sieberiana & 2,49 & Acacia senegal & 12,91 \\
\hline & Combretum micranthum & 2,49 & Combretum nigricans & 12,88 \\
\hline & Combretum Paniculatum & 2,41 & Cassia sieberiana & 11,97 \\
\hline & Cassia sieberiana & 1,9 & Albizia chevalieri & 7,49 \\
\hline \multirow{10}{*}{ Intermédiaire } & Mitagyna inermis & 157,32 & Balanites aegyptiaca & 54,18 \\
\hline & Piliostigma reticulatum & 25,8 & Piliostigma reticulatum & 45,28 \\
\hline & Diospyros mespiliformis & 12,56 & Mitagyna inermis & 44,25 \\
\hline & Daniellia oliverii & 7,62 & Diospyros mespiliformis & 37,02 \\
\hline & Acacia sieberiana & 6,66 & Combretum aculeatum & 13,31 \\
\hline & Rytigynia senegalensis & 6,14 & Anogeissus leiocarpus & 10,79 \\
\hline & Keetia cornelia & 6 & Ziziphus mauritiana & 8,93 \\
\hline & Morelia senegalensis & 5,82 & Pseudocedrela kotchyi & 8,77 \\
\hline & Acacia polyacantha & 5,76 & Crossopteryx febrifuga & 6,23 \\
\hline & Acacia ataxacantha & 5,72 & Tamarindus indica & 5,56 \\
\hline \multirow{10}{*}{ Aval } & Mitragyna inermis & 126,08 & Combretum glutinosum & 42,12 \\
\hline & Disopyros mespiliformis & 29,05 & Disopyros mespiliformis & 38,27 \\
\hline & Morelia senegalensis & 23,42 & Balanites aegyptiaca & 36,89 \\
\hline & Piliostigma reticulatum & 21,52 & Combretum micranthum & 26,65 \\
\hline & Feretia apodanthera & 18,13 & Combretum nigricans & 26,32 \\
\hline & Tamarindus indica & 15,12 & Piliostigma reticulatum & 23,13 \\
\hline & Balanites aegyptiaca & 7,51 & Daniella oliveri & 22,63 \\
\hline & Ziziphus mauritiana & 7,03 & Mitragyna inermis & 17,09 \\
\hline & Guiera senegalensis & 6,7 & Guiera senegalensis & 13,44 \\
\hline & Anogeissus leiocarpa & 6,06 & Tamarindus indica & 13,07 \\
\hline
\end{tabular}


Tableau 4 : Valeur d'Importance des Familles (FIV) selon les types de formations et les positions d'écoulement le long de la Sirba.

\begin{tabular}{lcccccc}
\hline \multirow{2}{*}{ Étiquettes de lignes } & \multicolumn{3}{c}{ Formation ripicole } & \multicolumn{4}{c}{ Savane } \\
\cline { 2 - 7 } & Amont & Aval & Intermédiaire & Amont & Aval & Intermédiaire \\
\hline Anacardiaceae & & & & 17,41 & & \\
Annonaceae & & & & 4,54 & & \\
Asclepiadaceae & 4,31 & 3,51 & 3,39 & 0,00 & 0,00 & 0,00 \\
Balanitaceae & 4,22 & 8,22 & 5,09 & 57,54 & 38,28 & 53,30 \\
Bignoniaceae & 0,00 & 0,00 & 0,00 & 4,18 & 0,00 & 0,00 \\
Capparaceae & 5,04 & 3,51 & 6,95 & 0,00 & 5,49 & 0,00 \\
Celastraceae & 0,00 & 0,00 & 0,00 & 0,00 & 0,00 & 3,39 \\
Combretaceae & 18,41 & 23,98 & 8,63 & 33,70 & 116,74 & 37,82 \\
Ebenaceae & 9,89 & 31,95 & 14,74 & 20,14 & 42,53 & 29,59 \\
Euphorbiaceae & 8,59 & 7,29 & 10,71 & 0,00 & 0,00 & 4,46 \\
Hippocrateaceae & 4,32 & 3,57 & 0,00 & 0,00 & 0,00 & 0,00 \\
Fabaceae-Caesalpinoideae & 23,21 & 38,96 & 38,41 & 37,13 & 32,01 & 47,68 \\
Fabaceae-Mimosoideae & 18,12 & 17,13 & 18,62 & 45,83 & 27,44 & 36,70 \\
Fabaceae-Faboideae & 0,00 & 3,63 & 3,38 & 0,00 & 0,00 & 7,42 \\
Loganiaceae & 0,00 & 0,00 & 0,00 & 4,16 & 0,00 & 0,00 \\
Malvaceae & 0,00 & 0,00 & 0,00 & 12,83 & 0,00 & 0,00 \\
Meliaceae & 0,00 & 0,00 & 3,56 & 0,00 & 12,13 & 9,76 \\
Moraceae & 4,45 & 0,00 & 0,00 & 0,00 & 0,00 & 3,57 \\
Olacaceae & 0,00 & 3,82 & 0,00 & 0,00 & 0,00 & 0,00 \\
Rhamnaceae & 4,48 & 10,79 & 8,02 & 9,71 & 11,88 & 14,31 \\
Rubiaceae & 185,62 & 139,34 & 166,12 & 0,00 & 13,50 & 48,41 \\
Sapindaceae & 0,00 & 4,31 & 0,00 & 0,00 & 0,00 & 0,00 \\
Sapotaceae & 5,14 & 0,00 & 0,00 & 52,86 & 0,00 & 0,00 \\
Sterculiaceae & 0,00 & 0,00 & 4,49 & 0,00 & 0,00 & 0,00 \\
Verbenaceae & 0,00 & 0,00 & 3,92 & 0,00 & 0,00 & 3,59 \\
\hline & & & & & &
\end{tabular}

Tableau 5 : Indice de similarité des ligneux adultes entre cordons ripicoles et entre les savanes suivant trois positions d'écoulement le long de la Sirba.

\begin{tabular}{llcccc}
\hline Sites & & \multicolumn{2}{c}{ Amont } & \multicolumn{2}{c}{ Zone intermédiaire } \\
\cline { 3 - 6 } & & Cordon & Savane & Cordon & Savane \\
\hline Amont & Cordon & & & & \\
& Savane & & & & \\
\hline \multirow{2}{*}{ Zone intermédiaire } & Cordon & 0,566 & 0,5 & & \\
& Savane & 0,6522 & 0,6772 & & \\
\hline Aval & Cordon & 0,653 & 0,545 & 0,561 & 0,615 \\
& Savane & 0,6364 & 0,6154 & 0,7083 & 0,6071 \\
\hline
\end{tabular}


Tableau 6: Caractéristiques structurales des trois cordons ripicoles et des savanes adjacentes suivant trois positions d'écoulement.

\begin{tabular}{lcccc}
\hline Position topologique & Type de formation & Nombre de relevés & Densité & Surface terrière \\
\hline \multirow{2}{*}{ Amont } & Cordon ripicole & 52 & $1226,92 \pm 82,94$ & $26,23 \pm 2,29$ \\
& Savane & 34 & $107,35 \pm 26,33$ & $2,41 \pm 0,57$ \\
\hline Intermédiaire & Cordon ripicole & 41 & $1067,80 \pm 93,47$ & $24,66 \pm 2,58$ \\
& Savane & 21 & $232,38 \pm 33,50$ & $7,43 \pm 0,73$ \\
\hline \multirow{2}{*}{ Aval } & Cordon ripicole & 47 & $765,11 \pm 87,26$ & $31,07 \pm 2,41$ \\
& Savane & 26 & $198,85 \pm 30,11$ & $4,85 \pm 0,65$ \\
\hline
\end{tabular}

Tableau 7 : Corrélation entre la densité, la richesse spécifique moyenne et les dimensions de la rivière.

\begin{tabular}{lccccccccc}
\hline Dimensions & & Densité & \multicolumn{4}{c}{$\begin{array}{c}\text { Richesse Spécifique } \\
\text { moyenne }\end{array}$} \\
\cline { 2 - 10 } & Amont & Inter & Aval & Amont & Inter & Aval & Amont & Inter & Aval \\
\hline Largeur & 0,034 & 0,293 & 0,037 & 0,102 & 0,292 & 0,044 & $14,63 \pm 2,05$ & $25,36 \pm 1,28$ & $29,33 \pm 1,33$ \\
Profondeur & 0,013 & 0,300 & 0,065 & 0,032 & 0,093 & 0,151 & $1,33 \pm 0,11$ & $3,89 \pm 0,22$ & $2,89 \pm 0,15$ \\
\hline \multicolumn{2}{c}{ Inter : Zone intermédiaire } & & & & & & &
\end{tabular}

Tableau 8 : Répartition des espèces dans les trois sites.

\begin{tabular}{|c|c|c|c|c|c|c|c|}
\hline \multirow[t]{2}{*}{ Espèces présentes } & \multirow[t]{2}{*}{ Familles } & \multicolumn{2}{|c|}{ Amont } & \multicolumn{2}{|c|}{$\begin{array}{c}\text { Zone } \\
\text { ntermédiaire }\end{array}$} & \multicolumn{2}{|c|}{ Aval } \\
\hline & & CR & $\mathbf{S}$ & $\mathbf{C R}$ & $\mathbf{S}$ & $\mathbf{C R}$ & $\mathbf{S}$ \\
\hline Acacia ataxacantha $D C$. & Leguminoseae-Mimosaceae & & & + & & + & \\
\hline Acacia gourmaensis A. Chev. & Leguminoseae-Mimosaceae & & + & & + & & \\
\hline Acacia hockii De Wild. & Leguminoseae-Mimosaceae & & + & & & & \\
\hline $\begin{array}{l}\text { Acacia macrostachya Reichenb. } \\
\text { ex. Benth. }\end{array}$ & Leguminoseae-Mimosaceae & & + & & & & \\
\hline Acacia nilotica (L.)Willd ex.Del & Leguminoseae-Mimosaceae & & + & & + & + & + \\
\hline Acacia pennata (L.) Willd. & Leguminoseae-Mimosaceae & + & + & + & + & + & + \\
\hline $\begin{array}{l}\text { Acacia polyacantha Willd. subsp. } \\
\text { campylacantha (Hoechst. ex A. Rich.) } \\
\text { Brenan }\end{array}$ & Leguminoseae-Mimosaceae & & & + & + & & \\
\hline Acacia sieberiana $D C$ & Leguminoseae-Mimosaceae & + & + & + & & + & \\
\hline Acacia senegal (L.) Willd & Leguminoseae-Mimosaceae & & + & & + & + & + \\
\hline Acacia seyal Del. & Leguminoseae-Mimosaceae & + & + & + & + & + & + \\
\hline Adansonia digitata $L$. & Bombacaceae & & + & & & & \\
\hline Albizia chevalieri Harms & Mimosaceae & + & + & + & + & + & + \\
\hline Ampelocissus leonensis (Hook. f.) Planch. & Vitaceae & & + & + & & & + \\
\hline Andira inermis (Wright) DC. & Fabaceae & & & & + & + & \\
\hline Annona senegalensis Pers. & Annonaceae & & + & & & & \\
\hline $\begin{array}{l}\text { Anogeissus leiocarpus (DC.) Guill. \& Perr. } \\
\text { Antidesma venosum Tul. }\end{array}$ & $\begin{array}{l}\text { Combretaceae } \\
\text { Euphorbiaceae }\end{array}$ & + & + & $\begin{array}{l}+ \\
+\end{array}$ & + & $\begin{array}{l}+ \\
+\end{array}$ & + \\
\hline
\end{tabular}




\begin{tabular}{|c|c|c|c|c|c|c|c|}
\hline Asparagus africanus Lam. & Liliaceae & & + & & & & \\
\hline Azadirachta indica A. Juss. & Meliaceae & + & + & & & & \\
\hline Balanites aegyptiaca (L.) Del. & Zigophyllaceae & + & + & + & + & + & + \\
\hline Bauhinia rufescens Lam. & $\begin{array}{l}\text { Leguminoseae- } \\
\text { Caesalpiniaceae }\end{array}$ & & & & & & + \\
\hline Boscia angoustifolia A. Rich. & Capparaceae & & + & & & & + \\
\hline Boscia senegalensis (Pers.) Lam. ex Poir. & Capparaceae & & & & & & + \\
\hline Bridelia sclereunera Müll. Arg. & Euphorbiaceae & + & & & & & \\
\hline Calotropis procera (Ait.) Ait.f. & Asclepiadaceae & & + & & + & & \\
\hline $\begin{array}{l}\text { Capparis sepiaria L. var. fisheri (Pax) De } \\
\text { Wolf }\end{array}$ & Capparaceae & & + & & + & & + \\
\hline Capparis tomentosa Lam. & Capparaceae & + & + & + & & + & + \\
\hline Caralluma dalzielii N.E. Br. & Asclepiadaceae & & + & & & & + \\
\hline Caralluma decaisniana (Lem.) N.E. Br. & Asclepiadaceae & & + & & + & & \\
\hline Cassia sieberiana $D C$ & $\begin{array}{l}\text { Leguminoseae- } \\
\text { Caesalpiniaceae }\end{array}$ & + & + & + & + & + & \\
\hline Cassia singueana Del. & $\begin{array}{l}\text { Leguminoseae- } \\
\text { Caesalpiniaceae }\end{array}$ & + & + & & & & \\
\hline Cissus quadrangularis $L$. & Vitaceae & & & & & + & + \\
\hline Cola laurifolia Mast. & Rubiaceae & & & + & & & \\
\hline Combretum aculeatum Vent. & Combretaceae & + & + & + & + & + & + \\
\hline Combretum glutinosum Perr. ex DC. & Combretaceae & + & + & & + & + & + \\
\hline Combretum fragrans $F$. Hoffm. & Combretaceae & & & & + & & \\
\hline Combretum micranthum G. Don & Combretaceae & + & + & + & + & + & + \\
\hline $\begin{array}{l}\text { Combretum nigricans Lepr. ex Guill. \& } \\
\text { Perr. }\end{array}$ & Combretaceae & & + & + & + & & + \\
\hline Combretum paniculatum Vent. & Combretaceae & + & + & + & + & + & \\
\hline Commiphora africana (A. Rich.) Engl. & Burseraceae & & + & & + & & + \\
\hline Crateva adansonii $D C$ & Capparaceae & + & + & + & + & + & \\
\hline $\begin{array}{l}\text { Crossopteryx febrifuga (Afz. ex G. Don) } \\
\text { Benth. }\end{array}$ & Rubiaceae & & + & & + & & \\
\hline Dicrostachys cinerea (L.) Wight \& Arn. & Mimosaceae & + & + & + & + & & \\
\hline Daniellia oliveri (Rolfe) Hutch. \& Dalz. & caesalpiniaceae & + & & + & + & + & + \\
\hline Desmodium velutinum (Willd.) DC. & $\begin{array}{l}\text { Leguminoseae- } \\
\text { Papillonaceae }\end{array}$ & + & & & & & \\
\hline $\begin{array}{l}\text { Diospyros mespiliformis Hochst. ex A. DC. } \\
\text { Euphorbia sp }\end{array}$ & Ebenaceae & + & $\begin{array}{l}+ \\
+ \\
+\end{array}$ & + & + & + & + \\
\hline Feretia apodanthera Del. & Rubiaceae & + & + & + & + & + & + \\
\hline Ficus platiphylla Delile & Moraceae & + & & + & + & & \\
\hline Ficus thonningii Blume & Moraceae & + & & & & & \\
\hline $\begin{array}{l}\text { Ficus sycomorus L. subsp. gnaphalocarpa } \\
\text { (Miq.) C.C. Berg }\end{array}$ & Moraceae & & & + & + & & \\
\hline Fluggea virosa (Roxb. ex Willd.) Voigt & Euphorbiaceae & + & + & + & + & + & + \\
\hline Gardenia erubescens Stapf \& Hutch. & Rubiaceae & & + & & & & \\
\hline Gardenia ternifolia Schum. \& Thonn. & Rubiaceae & + & + & + & + & + & + \\
\hline Gardenia sokotensis Hutch. & Rubiaceae & & + & & & & + \\
\hline Grewia bicolor Juss. & Tiliaceae & & & + & & & \\
\hline Grewia flavescens Juss. & Tiliaceae & & + & & + & + & + \\
\hline Grewia lasiodiscus K. Schum. & Tiliaceae & & + & + & + & + & + \\
\hline Guiera senegalensis J.F. Gmel. & Combretaceae & + & + & + & + & + & + \\
\hline Hollarrhena floribunda A. CD. & Apocynaceae & & + & & & & \\
\hline Gymnema sylvestris (Retz.) Schultes & Asclepiadaceae & + & & + & + & + & + \\
\hline Jasminium obtusifolium Bak. & Oleaceae & & & & & + & \\
\hline Khaya senegalensis (Desv.) A. Juss. & Méliaceae & & + & & & + & + \\
\hline
\end{tabular}




\begin{tabular}{|c|c|c|c|c|c|c|c|}
\hline Keetia cornelia (Cham. Et Sclecht.) & Rubiaceae & & & + & & & \\
\hline Keetia venosa (Oliver) & Rubiaceae & & & + & & & \\
\hline Kigelia africana (Lam.) Benth. & Bignoniaceae & & & & & & + \\
\hline Lannea acida A. Rich. & Anacardiaceae & & + & & & & \\
\hline Lannea microcarpa Engl. \& K. Krause & Anacardiaceae & & + & & & & \\
\hline $\begin{array}{l}\text { Loeseneriella africana (Willd.) Wilczeck ex } \\
\text { Hallé }\end{array}$ & Hyppocrataceae & + & & & & + & + \\
\hline Maytenus senegalensis (Lam.) Exell & Celastraceae & & + & + & + & & + \\
\hline Mimosa pigra $L$. & Mimosaceae & & & + & & + & \\
\hline Mitragyna inermis (Willd.) O. Ktze. & Rubiaceae & + & + & + & & + & + \\
\hline Morelia senegalensis A. Rich. ex DC. & Rubiaceae & & & + & & + & \\
\hline Paullinia pinnata $L$ & Sapindaceae & & & + & & + & + \\
\hline Phyllanthus reticulatus Poir. & Euphorbiaceae & + & + & + & & + & \\
\hline Piliostigma reticulatum (DC.) Hochst. & $\begin{array}{l}\text { Leguminoseae- } \\
\text { Caesalpiniaceae }\end{array}$ & + & + & + & + & + & + \\
\hline $\begin{array}{l}\text { Piliostigma thonningii (Schum.) Milne- } \\
\text { Redhead }\end{array}$ & $\begin{array}{l}\text { Leguminoseae- } \\
\text { Caesalpiniaceae }\end{array}$ & + & & + & + & & \\
\hline Pterocarpus erinaceus Poir. & $\begin{array}{l}\text { Leguminoseae- } \\
\text { Papillonaceae }\end{array}$ & & + & & + & & \\
\hline Pteleopsis suberosa Engl. \& Diels & Combretaceae & & + & & & & \\
\hline Prosopis africana (Guill. \& Perr.) Taub. & Mimosaceae & & & & & + & + \\
\hline Pseudocedrela kotchyi (Schweinf.) Harms & Méliaceae & & & + & + & & \\
\hline Rytigynia senegalensis Blume & Rubiaceae & + & + & + & + & + & + \\
\hline Sclerocarya birrea (A. Rich.) Hochst. & Anacardiaceae & & + & & & & + \\
\hline Sterculia setigera Del. & Sterculiaceae & & + & & & & \\
\hline Stereospermum kuntianum Cham. & Bignoniaceae & & + & + & + & & + \\
\hline Strophanthus sarmenthosus DC. & Apocynaceae & & & & + & & \\
\hline Strychnos spinosa Lam. & Loganiaceae & & + & & & & \\
\hline Taccazea apiculata Oliv. & Asclepiaceae & + & & + & + & + & + \\
\hline Tamarindus indica $L$. & $\begin{array}{l}\text { Leguminoseae- } \\
\text { Caesalpiniaceae }\end{array}$ & & & & + & + & + \\
\hline Vitellaria paradoxa C.F. Gaertn. & Sapotaceae & + & + & & & & \\
\hline Vitex doniana Sweet & Verbenacae & + & & + & + & & \\
\hline Ximenia americana $L$ & Olacaceae & & + & & & + & \\
\hline Ziziphus abyssinica A. Rich. & Rhamnaceae & & & + & & + & \\
\hline Ziziphus mauritiana Lam. & Rhamnaceae & + & + & + & + & + & + \\
\hline Ziziphus mucronata Willd. & Rhamnaceae & + & + & + & + & + & + \\
\hline $\begin{array}{l}\text { Ziziphus spina-christi (L.) Desf. var. } \\
\text { microphylla Hochst. ex A. Rich. }\end{array}$ & Rhamnaceae & & - & + & & + & \\
\hline
\end{tabular}

$\mathrm{CR}$ : Cordon ripicole ; $\mathrm{S}$ : Savane

\section{DISCUSSION}

\section{Diversité taxonomique}

La flore ligneuse ripicole recensée dans cette étude représente $3,39 \%$ de la flore nationale. Elle est le double de la richesse floristique au niveau des cours d'eau temporaires recensée par Sambaré et al. (2011) au niveau du sahel strict.

Les cordons ripicoles de la Sirba sont dominées par l'espèce Mitragyna inermis et le même constat a été fait par Fontès et Guinko (1995) au niveau de la zone sahélienne du pays où les berges des cours d'eau sont occupées par des cordons ripicoles dominées par cette espèce. C'est une espèce caractéristique des cours d'eau temporaires dans le climat soudano-sahélien (Poilecot et al., 2009 ; Sambaré et al., 2011) mais aussi de certaines rivières du secteur sud soudanien (Bélem, 2008). L'Indice de Valeur 
d'Importance (IVI) de Mitragyna inermis montre en amont, dans la zone intermédiaire et en aval sa grande importance écologique dans les cordons ripicoles. Son IVI en amont $(220,84)$ est voisin de celui rapporté en zone soudanienne par Savadogo et al. (2007) qui est de (214,5). Elle est associée à Piliostigma reticulatum et Diospyros mespiliformis. Selon Sambaré et al. (2011), ce sont ces deux espèces qui sont dominantes le long des cours d'eau temporaires. La famille des Rubiaceae est prédominante avec une FIV de 185,6 en amont du cours d'eau. La présence des Rubiaceae étant liée à l'humidité des sols (Ouédraogo, 2006 ; Bognounou et al., 2009), il existe une logique au regard de la position amont de la rivière. En effet, elle subit une importante inondation due à la localisation d'un barrage en amont de ce site. Cependant, le pourcentage relativement faible des espèces de cette famille dans les berges montre une durée d'inondation faible en général. Malgré l'humidité des sols des cordons ripicoles leur richesse spécifique est significativement inférieure à celle des savanes adjacentes. Selon Savadogo et al., (2007), cette situation serait due à une densité très forte des espèces dominantes dans les galeries forestières qui ne favorise pas la germination d'autres espèces ainsi que le broutage et le piétinement de la régénération par les animaux.

Le recouvrement plus important dans les cordons ripicoles diminue la régénération à cause de l'effet d'ombrage et de la compétition. L'inondation prolongée des berges (Sambaré et al., 2010) serait aussi une des causes de la faible diversité des cordons ripicoles due aux conditions asphyxiantes. Selon Hien et al. (1995) l'élargissement du lit provoque le recul des berges et par la même occasion la baisse de la diversité végétale. En effet, la pratique des cultures maraichères étendue de la berge jusqu'au lit surtout dans la zone intermédiaire du fleuve cause un ensablement important du lit et une perte de certaines espèces. Au niveau de la Sirba, la pression anthropique sur les berges provoque une perte de la diversité végétale. Les principaux facteurs de perturbation sont les cultures du tabac, de la pastèque, du haricot, les activités minières, l'exploitation anarchique du bois et la présence de barrage. Certains auteurs (Natta 2003 ; Braatne et al., 2007 ; Kokou et al., 2008) ont reconnu ces actions anthropiques comme étant les principales menaces de la végétation des berges. Une des conséquences des perturbations est la fréquence des zones nues le long du bassin versant de la Sirba. Cette dégradation du couvert végétal est le point de départ du processus de dégradation des sols (Hien et al., 1995).

\section{Similarité et diversité spécifique}

Nos résultats sur la richesse floristique des berges ne sont pas en accord avec ceux de Sambaré et al. (2010) et (2011). Ainsi, selon cet auteur la richesse spécifique augmente du nord sahélien vers le sud soudanien. Dans cette logique, l'amont appartenant au secteur phytogéographique nord soudanien contrairement aux deux autres positions qui appartiennent à celui sub-sahélien devrait être la plus diversifiée. Cependant, cette partie du cours d'eau a le lit mineur le plus étroit (14,63 $\mathrm{m})$ et la plus faible profondeur $(1,33 \mathrm{~m})$ due à l'ensablement. Il n'y a aucune corrélation entre la largeur, la diversité et la densité dans les trois sites. Cela est sans doute liée à la fragmentation très poussée des cordons ripicoles. La présence d'un barrage en amont de ce site expliquerait sa faible diversité. En effet, la construction des barrages le long des rivières fournit l'impact anthropique dominant sur l'environnement de la rivière surtout à l'aval du barrage (Nilsson et al., 2005 ; Braatne et al., 2007). Avec l'endiguement, la zone inondée subit en amont une transition brusque, dramatique de la vallée fluviale au réservoir (Nilsson et Berggren 2000; Naiman 
et al., 2005). Les conséquences écologiques sur la partie aval des barrages sont un changement de la quantité d'eau et le temps de flux, la réduction des matériaux alluviaux et particulièrement des sédiments (Magilligan et al., 2003) et la fragmentation des corridors de la rivière. Ainsi, l'écoulement de l'eau n'est possible qu'après le remplissage du barrage et l'inondation perdure éliminant toute espèce non hygrophyle ce qui réduit la richesse spécifique. En effet, selon Natta (2003) la présence des cordons ripicoles s'explique par la dynamique fluviale incluant l'inondation et le transport des sédiments

La zone intermédiaire du cours d'eau est la plus diversifiée. Ainsi, la partie intermédiaire se comporte comme une zone temporairement inondable qui renferme les cordons ripicoles les plus diversifiées (Sambaré et al., 2010). Elle possède une espèce qui est absente dans les deux autres positions d'écoulement. Il s'agit de Cola laurifolia qui est le plus souvent rencontrée au niveau des cours d'eau semi-permanents et permanents (Sambaré et al., 2011). Cette espèce est selon Mugnier (2008) rencontrée au Burkina Faso au niveau des galeries forestières dans les zones soudano-guinéennes et guinéennes. La présence de $C$. laurifolia témoigne des conditions hydriques jadis bonnes. Elle a été rencontrée dans un vestige de bois sacré le long du cours d'eau temporaire Nakanbé dans la même zone sudsahélienne du Burkina Faso (Sambaré, 2013) ce qui témoigne de sa disparition due à l'action anthropique. Acacia ataxacantha, Antidesma venosum et Pseudocedrela kotschyi sont également des espèces qui ne se rencontrent que dans la zone intermédiaire. Si les deux premières sont généralement rencontrées au bord des cours d'eau où l'humidité est moyenne la dernière, elle, est une espèce de savanes sur sol lourd mal drainé (Mugnier, 2008). Pseudocedrela kotchyi est une espèce très utilisée dans la pharmacopée dans cette zone. Bien qu'elle soit une espèce de savane elle ne se rencontre qu'au niveau des berges sur nos sites qui jouent donc un rôle refuge.

La grande similarité rencontrée entre les cordons ripicoles et la savane adjacente montre le caractère temporaire de l'écoulement au niveau de la rivière. Ainsi, l'écoulement dure une partie de la saison pluvieuse puis pendant la saison sèche les berges offrent des conditions favorables à l'installation des espèces savanicoles par rapport aux milieux environnants. La ressemblance entre la composition floristique de la zone intermédiaire et l'aval de la rivière est due à une durée d'inondation presque similaire de ces deux sites. La zone intermédiaire étant le site de transit des eaux, elle a la période de crue la plus longue. En effet, cette zone a une faible altitude par rapport à l'amont et à l'aval ce qui favorise l'inondation des berges créant ainsi de bonnes conditions pour les espèces de Rubiaceae.

\section{Structure}

La distribution générale en classes de diamètre des individus des cordons ripicoles et des savanes adjacentes montre dans les trois sites des paramètres de forme (c) inférieurs ou proche de 1 avec une tendance en "J renversé". Ces formations ont des peuplements relativement stables avec une distribution multispécifique car possèdant plus de pieds de petit diamètre (Ryniker et al., 2006). Plusieurs auteurs considèrent cette distribution comme celle de peuplements capables de recruter des individus dans le temps, donc stables (Natta 2003; Zegeze et al., 2006 ; Sambaré et al., 2011). Ce type de distribution révèle un bon potentiel de régénération de la population (Zegeze et al., 2006). Les résultats de la présente étude sont similaires à ceux de Sambaré et al. (2011) qui rapportent les mêmes structures au niveau des rivières temporaires du Burkina Faso. Les 
cordons ripicoles sont semi-décidues donc sont broutés pendant les périodes sèches (Savadogo et al., 2007) et cela explique les faibles effectifs de la première classe des arbres et de la régénération. En effet, on note une difficulté de recrutement des individus juvéniles des classes de hauteur inférieure vers celles supérieures. Il y a donc une possibilité de vieillissement de ces formations. Cette végétation bien que jeune est très confinée et fragmentée. Le paramètre de forme de la distribution en aval très proche de 1 sous-entend un peuplement ripicole vieillissant au niveau de l'aval de la Sirba avec une part de $4,06 \%$ d'individus de gros diamètre $(>40 \mathrm{~cm})$ et les individus juvéniles sont cantonnées dans les deux premières classes de hauteur.

Les densités des cordons ripicoles sont trois fois supérieures à celles des savanes adjacentes, s'expliquant par les conditions d'humidité favorables aux niveaux des berges. En effet, la disponibilité de l'eau pendant la saison pluvieuse et la proximité de la nappe phréatique pendant la saison sèche au niveau de la berge est un atout pour l'installation des plantes en zone aride. Des études antérieures (Sambaré et al., 2011) ont révélé des densités plus faibles des peuplements par rapport à nos résultats au niveau des rivières temporaires du Burkina Faso. Ces données appartiennent à plusieurs rivières temporaires qui n'ont pas forcement les mêmes paramètres et situées dans le secteur phytogéographique sahel strict. Ces densités décroissent de l'amont vers l'aval du fleuve avec une différence significative $(\mathrm{P}<0,001)$ entre les densités des cordons ripicoles en amont et en aval. La faible densité des cordons ripicoles en aval de la rivière s'explique par la pression anthropique plus élevée. Dans la zone sahélienne du Burkina Faso, Mitragyna inermis est une espèce de choix dans la construction d'habitats (Ganaba et al., 2004) et la sollicitation est fonction des groupes ethniques. Cela constitue une source de pression sur elle en plus de l'élevage. Les formations à $M$. inermis régressent également au profit de la culture de la pastèque et du tabac. En effet, les ressources dont l'eau, le poisson, l'humidité et la fertilité des sols amènent les populations riveraines au cours d'eau à les utiliser de façon excessive causant ainsi la fragmentation des cordons ripicoles et l'ensablement des lits mineurs.

Les densités des savanes adjacentes sont relativement plus faibles et cela est dû, à l'exploitation agricole des terres voisines des berges. Cette pression dégrade les savanes adjacentes laissant les cordons ripicoles sans protection pour la plupart. Les savanes en amont possèdent la plus faible densité de peuplement malgré leur appartenance au climat nord soudanien, relativement plus arrosé et avec une plus forte diversité végétale. Cela s'explique par une plus grande pression d'exploitation des savanes en amont. L'agriculture est la principale activité en amont du fleuve et les zones de prédilections sont les berges.

Les surfaces terrières des cordons ripicoles révèlent une importante quantité de bois par rapport à la savane adjacente. Selon Fontès et Guinko (1995) la surface terrière moyenne rencontrée en zone sahélienne et considéré comme forte est de l'ordre de 2,5 à $8,75 \mathrm{~m}^{2} / \mathrm{ha}$. Nos résultats montrent des surfaces terrières moyennes des trois cordons ripicoles $\left(27,21 \mathrm{~m}^{2} / \mathrm{ha}\right)$ supérieures à celle rencontrée dans les rivières temporaires du secteur sud-sahélien du Burkina Faso (14,22 $\mathrm{m}^{2} /$ ha) par Sambaré et al. (2011), révélant un potentiel important en bois au niveau des cordons ripicoles de la Sirba. Ce potentiel montre un important stock de biomasse au niveau des berges, d'où un important stock de carbone. Les cordons ripicoles pourraient donc constituer des sites de prédilection pour la séquestration de carbone atmosphérique. 


\section{Conclusion}

Cette étude a permis de mettre en évidence un très bon potentiel en bois au niveau des berges de la Sirba. La faible diversité des cordons ripicoles de la Sirba par rapport à celle des savanes adjacentes témoigne d'une pression anthropique intense sur ce cours d'eau. Cependant, malgré la faible diversité des cordons ripicoles par rapport aux savanes, la rivière renferment des espèces rares au sahel Cola laurifolia, Morelia senegalensis et Keetia venosa ce qui enrichit la flore de la localité. Les cordons ripicoles sont stables avec un assez bon recrutement des individus dans les différentes classes de diamètre mais une dynamique régressive est attendue vu le faible taux des juvéniles. Quant aux savanes, elles sont en disparition autour de la rivière au profit des champs cultivés. Nous pouvons considérer cette formation comme un bon potentiel de séquestration du carbone atmosphérique au regard de l'importance de la quantité de bois qui s'y trouve. Ces formations constitueraient donc des sites de séquestration importante de carbone qui peuvent susciter un crédit pour le piégeage du carbone atmosphérique par le reboisement au profit des berges et de la population locale.

\section{REFERENCES}

Braatne JH, Rood ST, Goater LA, Blair CL. 2007. Analyzing the impacts of Dams on riparian ecosystems: A review of research strategies and their relevance to the snake River through Hells Canyon. Environmental Management, 41(4): 267281.

Belem OM. 2008, Les galeries forestières de la Réserve de la Biosphère de la Mare aux Hippopotames du Burkina Faso : caractéristiques, dynamique et ethnobotanique. Thèse de doctorat d'état, Université de Ouagadougou, p. 279.
Bognounou F, Thiombiano A, Savadogo P, Boussim JI, Oden PC, Guinko S. 2009, Woody vegetation structure and composition at four sites along a latitudinal gradient in Western Burkina Faso. Bois et Forets des Tropiques, 300(2): 30-44.

Crawley MJ. 2005. Statistics: An Introduction Using, Chister R (ed). John Wiley \& Sons: England.

Cecchi P, Meunier-Nikiema A, Moiroux N, Sanou B, Bougaire F. 2007. Why an Atlas of Lakes and Reservoir in Burkina Faso? AfricaGIS : Ouagadougou ; 1-20.

Dipama JM. 2010. Principaux facteurs environnementaux du Burkina Faso. In Atlas de la Biodiversité du Burkina Faso: le Milieu Biophysique, Thiombiano, Kapmann (eds). University Press : Franckfurt ; 122-134.

Fontes J, Guinko S. 1995. Carte de la Végétation et de l'Occupation des Sols du Burkina Faso. Notice explicative, Ministère de la Coopération français, projet Campus, Toulouse, $68 \mathrm{p}$.

Ganaba S, Ouadba JM, Bognounou O. 2004, Plantes de construction d'habitats en région sahélienne. Bois et Forêts des Tropiques, 282(4): 11-17.

Guinko S. 1984. Végétation de la Haute Volta. Thèse de Doctorat Es Sciences naturelles, Université de Bordeau III, France, tome 1, p. 318.

Henszey RJ, Skinner QD, Wesche TA. 1991. Response of montane meadow vegetation after two years of streamflow augmentation. Regulated Rivers: Research \& Management, 6: 29-38.

Hien F, Compaore JA, Coulibaly-Some O. 1995. La dynamique de la dégradation des sols dans le bassin du Nakambé : une étude diachronique dans le secteur des forêts classées de Bissiga-Nakabé au Burkina Faso, Colloques et Séminaires, 523-530. 
Hubble TCT, Docker BB, Rutherfurd ID. 2010. The role of riparian trees in maintaining riverbank stability: a review of Australian experience and practice. Ecologicam Engineering, 36: 292-304.

Kokou K, Adjossou K, Kokutse AD. 2008. Considering sacred and riverside forests in criteria and indicators of forest management in low wood producing countries: The case of Togo. Ecological Indicators, 8: 158-169.

Krebs C. 1999. Ecological Methodology. Benjamin/Cumings, Addison-Wesley Longman Educational Publishers: New York.

Ryniker KA, Bush JK, Van Auken OW. 2006. Structure of Quercus gambelii communities in the LincolnNational Forest, New Mexico, USA. Forest Ecology and Management, 233(1): 69-77.

Lavigne Delville MPh, Boucher L et Vidal L, 1996. Les bas-fonds en Afrique tropicale humide : stratégies paysannes, contraintes agronomiques et aménagements. In Fertilité du Milieu et Stratégies Paysannes sous les Tropiques Humides. actes du séminaire international, CIRAD : 148-161.

Magilligan FJ, Nislow KH, Graber BE. 2003. Scale-independent assessment of discharge reduction and riparian disconnectivity following flow regulation by dams. Geology, 31: 569-572.

Magurran AE. 2004. Measuring Biological Diversity. Blackwell Publishing: Malden, Oxford and Victoria; p. 256.

Morse JL, Megonical JP, Walbridge MR. 2003. Sediment nutrient accumulation and nutrient availability in two tidal freshwater marshes along the Mattaponi River, Virginia, USA. Biogeochemistry, 69: 175-206.

Mouchet F, Debruxelles N, Graux G, Dufays E, Augiron K, Claessens H. 2004. Physionomie et composition des zones riveraines des cours d'eau Wallonie. Forêt Wallonne, 68: 2-7.

Mugnier J. 2008. Nouvelle flore illustrée du Sénégal et des régionsvoisines, Jacques Mugnier. In Flore Illustrée du Sénégal (Tome 6). Copie électronique. Plantas del Sahara.

Naiman RJ, Decamps H, McClain ME, Likens GE. 2005. Riparia - Ecology, Conservation, and Management of Streamside Communities. Elsevier Acad. Pr: Amsterdam; 269-290.

Natta AK. 2003. Ecological assesment of riparian forests in Benin: phytodiversity, phytosociology and spatial distribution of trees species. PhD Thesis, Wageningen University, Netherlands, p. 213.

Nilsson C, Berggren K. 2000. Alteration of riparian ecosystems caused by river regulation. BioScience, 50: 783-792.

Nilsson C, Reidy CA, Dynesius M, Revenga C. 2005. Fragmentation and flow regulation of the world's large river systems. Science, 308: 405-408.

Monnier Y, 1990. La poussière et la Cendre. Paysages, Dynamique des Formations Végétales et Stratégies des Sociétés en Afrique de l'Ouest (2è édn). Min. Coopération et Agence de Coopération Culturelle et Technique : Paris ; 264.

Ouédraogo O, Thiombiano A, Hanhn-hadjali K, Guinko S. 2008. Diversité et structure des groupements ligneux du parc national d'Arly (Est du Burkina Faso). Flora et Vegetatio-Sudano-Sambesica, 11: 5-16.

Poff NL, Allan JD, Bain MB, Karr JR, Prestegaard KL, Richter BD, Sparks RE, Stromberg JC. 1997. The natural flow regime: a paradigm for river conservation and restoration. BioScience, 47: 769-784.

Poilecot P, Saidi S, N'Gakoutou EB. 2009, Phytogéographie du Parc national de Zakouma (Sud-Est du Tchad). Sécheresse, 20(3): 286-295. 
Sambaré O, Ouédraogo O, Wittig R, Thiombiano A. 2010. Diversité et écologie des groupements ligneux des cordons ripicoles du Burkina Faso (Afrique de l'Ouest). Int. J. Biol. Chem. Sci., 4(5): 1782-1800.

Sambaré O, Bognounou F, Wittig R, Thiombiano A. 2011, Woody species composition, diversity and structure of riparian forests of four watercourses types in Burkina Faso. Journal of Forestry Research, 22(2): 145-158.

Sambaré O. 2013. Diversité, phytosociologie et structures des cordons ripicoles du Burbina Faso. Thèse de doctorat unique de l'université de Ouagadougou, p. 224.

Savadogo P, Tigabu M, Sawadogo L, Odén PC. 2007. Woody species composition, structure and diversity of vegetation patches of a Sudanian savanna in Burkina
Faso. Bois et Forêts des Tropiques, 294(4): 5-20.

Sokpon, N, Sinadouwirou, TH, Gbaguidi, F, Biaou, H. 2001. Aperçu sur les forêts édaphiques hygrophiles du Benin. Belg. $J$. Bot., 134: 79-93.

Tremblay FM, Bergeron Y, Lalond D, Maufette Y. 2002. The sexual effects of potential reproduction and seedling recruitment on the maintenance of red maple (Acer rubrum L.) populations at the northern limit of the species range. Journal of Biogeography, 29: 365-373.

Zegeye HL, Teketay D, Kelbessa E. 2006. Diversity, regeneration status and socioeconomic importance of the vegetation in the islands of Lakes Ziway, South-central Ethiopia. Flor Morphol. Distribut. Funct. Ecol. Plants, 201: 483-498. 